\section{The state of public health in Canada}

The Public Health Agency of Canada recently released the "Report on the State of Public Health in Canada 2008", the first in a series of annual reports from the Chief Public Health Officer which will address the country's pressing public health challenges. This report is a must read for everyone who has an interest in the health and well-being of Canadians. Although it does not break new ground and most public health professionals should not learn much that they did not already know, the report does a superb job as an introduction to public health in Canada today (http://www.phac-aspc.gc.ca/publicat/ 2008/cpho-aspc/pdf/cpho-report-eng.pdf). It was obviously written with a much wider audience and a broader and longterm perspective in mind.

At less than 100 pages, it is concise enough and written in a very accessible style to be of interest to a general readership not familiar with public health. In particular, health professionals, decision- and policy-makers, NGOs, the media, the general public and elected officials should benefit from a careful study of the report. It defines public health in Canada, provides a brief history of its role in our country and describes major health indicators in comparison with other countries. Chapters 4 and 5 are probably the most important and describe with numerous examples and data the importance of social and economic factors and the challenges of health inequalities. The final chapter is a call to arms for all Canadians to work towards a healthier society through population-level efforts to reduce inequalities and create social, economic and environmental conditions which will support communities and individuals to make healthy choices and reduce hazards.

With over 400 references, clear logic and a good underlying conceptual framework, the report provides a rigorous approach to the documentation of health and social problems and it is authoritative in its discussion of potential solutions. It identifies several areas of needed research, illustrates succinctly key successes in policy and intervention and suggests general directions for policy, including: investments in families and children living in poverty; early child development programs; community capacity development; inter-sectoral actions; building the knowledge infrastructure - particularly to increase our understanding of subpopulations; and overall public health leadership. This report is a stepping stone. Not only does it provide a reference for future CPHO reports, it also defines a coherent vision and illustrates the core public health values which will be the basis for PHAC's leadership in public health in Canada for the years to come. All of us now need to disseminate this report to the widest possible audience. For one, it will now become required reading in my public health course for medical students at McGill.

Gilles Paradis

Interim Scientific Editor
LE MOT DE LA RÉDACTION

\section{La santé publique au Canada : état des lieux}

L'Agence de la santé publique du Canada a publié récemment le "Rapport sur l'état de la santé publique au Canada 2008 ", le premier d'une série de rapports annuels de l'administrateur en chef de la santé publique qui porteront sur les problèmes de santé publique les plus pressants au pays. Le rapport est un incontournable pour quiconque s'intéresse à la santé et au bien-être des Canadiens. Il n'ouvre pas de nouvelles avenues, et la plupart des professionnels de la santé publique n'y apprendront sans doute pas grand chose de nouveau, mais c'est une magnifique introduction à la santé publique telle qu'elle existe au Canada aujourd'hui (http://www.phac-aspc.gc.ca/publicat/2008/cphoaspc/pdf/cpho-report-fra.pdf). De toute évidence, il s'adresse à un auditoire beaucoup plus vaste et est rédigé dans une perspective générale et à long terme.

Concis (il fait moins de 100 pages) et rédigé dans un style très direct, le rapport intéressera les lecteurs ordinaires qui ne connaissent pas la santé publique. Les professionnels de la santé, les décideurs, les membres d'ONG, les journalistes, le grand public et les élus, surtout, gagneraient à l'étudier soigneusement. On y définit la santé publique, on y présente une brève histoire de son rôle au Canada, et on y compare les grands indicateurs de la santé au Canada et dans d'autres pays. Les chapitres 4 et 5 sont probablement les plus importants; truffés d'exemples et de chiffres, ils soulignent l'importance des facteurs socioéconomiques et les problèmes causés par les inégalités en santé. Le dernier chapitre appelle tous les Canadiens à lutter pour une société plus saine en appuyant la lutte contre les inégalités à l'échelle des populations et en créant les conditions sociales, économiques et environnementales qui incitent les gens, individuellement et collectivement, à prendre de saines habitudes de vie et à éviter de mettre la santé publique en danger.

Avec plus de 400 renvois, construit selon une structure logique et un cadre conceptuel solide, le rapport consigne rigoureusement les problèmes sociosanitaires et présente avec conviction les solutions possibles. Il indique plusieurs pistes de recherche, illustre brièvement les politiques et les mesures d'intervention exemplaires et donne des consignes générales : investir dans l'aide aux familles et aux enfants vivant sous le seuil de la pauvreté; investir dans le développement du jeune enfant; renforcer les capacités des collectivités; avoir une action intersectorielle; bâtir une infrastructure du savoir, en particulier pour mieux cerner les segments de la population; et exercer un rôle directeur en santé publique. Ce rapport est un point de départ. Non seulement constitue-t-il une référence pour les futurs rapports de l'administrateur en chef de la santé publique, mais il propose une vision cohérente et présente les valeurs fondamentales qui inspireront le leadership de l'ASPC en santé publique au Canada au cours des années à venir. À nous maintenant de le faire connaître au plus vaste auditoire possible. Pour ma part, je l'inscris à la liste de lecture de mon cours sur la santé publique pour les étudiants en médecine de l'Université McGill.

Le rédacteur scientifique intérimaire, Gilles Paradis 